\title{
Does the timing of antihypertensive agents affect clinical outcomes in patients with chronic kidney disease?
}

Taking at least one antihypertensive medication at bedtime is associated with a decreased risk of cardiovascular events in patients with chronic kidney disease (CKD) and hypertension, say researchers.

"A large number of published prospective trials have reported clinically meaningful morning-evening treatment time differences in blood pressure (BP)lowering efficacy, duration of action, safety profile, and/or effects on the circadian BP pattern for different classes of hypertension medications," explains corresponding author Ramón Hermida. "Independent trials have documented that ingesting at least one BP-lowering medication at bedtime, as compared to taking all medications upon wakening, is associated with increased BP control, a lowering of sleep-time BP, a decreased prevalence of nondipping, and a reduction in urinary protein excretion. We decided to take the investigations one step further and, to our knowledge, our study is the first to prospectively investigate the influence of time of day of hypertension treatment on cardiovascular risk."

\section{4 ... mean sleep-time BP} was lower among patients who took at least one medication at bedtime... 77

In their open-label trial, Hermida et al. randomly assigned 661 patients with hypertension and CKD to either take all of their hypertension medications on waking up or to take at least one of them at bedtime. The researchers found that, after a median follow-up of 5.4 years, patients who had been taking at least one antihypertensive medication at bedtime had a significantly lower risk of total cardiovascular morbidity or mortality (a composite of all-cause death, myocardial infarction, angina pectoris, coronary revascularization, heart failure, arterial occlusion of the lower extremities, occlusion of the retinal artery, and stroke) than patients who took all of their medications on waking up (adjusted hazard ratio 0.31 ). The reduction in the risk of major cardiovascular disease events (a composite of cardiovascular death, myocardial infarction, and stroke) was also significantly lower among patients who took at least one BP-lowering drug at bedtime. In addition, mean sleep-time $\mathrm{BP}$ was lower among patients who took at least one medication at bedtime, and more of these patients showed control of their ambulatory BP.

Rebecca Ireland

Original article Hermida, R. C. et al. Bedtime dosing of antihypertensive medications reduces cardiovascular risk in CKD. J. Am. Soc. Nephrol. doi:10.1681/ASN.2011040361 